\title{
Evaluación de la degradación por compactación de materiales granulares tipo subbase
}

\author{
Allex Eduardo Álvarez Lugo ${ }^{1}$ \\ $\triangle$ LEIDY VANESSA ESPINOSA RuIZ ${ }^{2}$ \\ PAOLA ANDRA ORTIZ RIVERA ${ }^{1}$ \\ MARLON DAVID HuRTADo RoBAYO ${ }^{3}$ \\ LEONEL EDUARdo Cotes De LA Hoz ${ }^{4}$ \\ YENIS MARGARITA LÓPEZ ESALAS ${ }^{1}$
}

\section{Resumen}

Los materiales granulares de subbase y base empleados en estructuras de pavimento son susceptibles a degradarse debido a múltiples factores inherentes a los procesos de producción, construcción y operación vial. El objetivo del presente artículo es cuantificar la degradación de materiales granulares por la incidencia de la recompactación, empleando para esto parámetros de caracterización física y mecánica. Esta degradación fue evaluada en términos de cambios en granulometría y respuesta mecánica (i.e., CBR, parámetros obtenidos del ensayo triaxial estático, y módulo resiliente). Los resultados obtenidos sugieren que la compactación sucesiva condujo a la producción de partículas finas, logrando un incremento del peso unitario seco, mientras se reduce la respuesta mecánica. Reducciones de CBR y módulo resiliente se evidencian desde la primera recompactación mostrando el efecto de la aplicación repetida de energía mecánica. Se recomienda investigación adicional para validar la relación entre la degradación inducida por recompactación y la generada en campo.

Palabras clave: agregados, subbase granular, degradación, relación de soporte de California (CBR), módulo resiliente, recompactación.

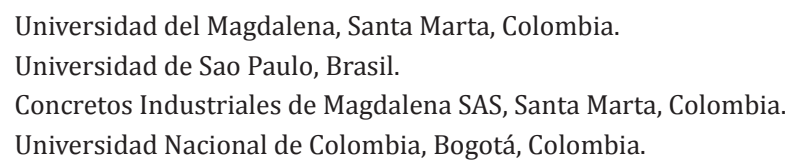

Autor de correspondencia: Espinosa Ruiz, L.V. (Leidy

Vanessa): Mz N casa 328 Villa Marbella, Santa Marta, Colombia. Teléfono: 3128381253.

Correo electrónico: leidy2418@hotmail.com
Historia del artículo:

Artículo recibido: 10-VIII-2015 / Aprobado: 28-XII-2018

Disponible online: 3 de febrero de 2019

Discusión abierta hasta octubre de 2020 


\section{Evaluation of degradation due to compaction in subbase granular materials}

\section{Abstract}

The granular bases and subbases used in pavement structures are materials susceptible to degrade due to multiple factors related to their production, construction, and operation as road materials. This paper aims on quantifying the degradation in materials used as granular subbase layers subjected to repeated compaction. This degradation was evaluated in terms of changes in gradation and mechanical response (i.e., CBR, parameters computed based on the static triaxial test, and resilient modulus). Corresponding results suggested that the recompaction process led to the production of fine particles, associated with the increase in the dry unit weight, while the mechanical response decreased. Reductions in CBR and resilient modulus were obtained since the first recompaction and showed the effect of applying repeated mechanical energy. Additional research is recommended to validate the relationship between the degradation induced by recompaction and that generated in the materials when used as pavement structural layers.

Keywords: aggregates, granular subbase, degradation, California bearing ratio (CBR), resilient modulus, recompaction.

\section{Avaliação da degradação pela compactação de materiais granulares, sub-base}

\section{Resumo}

Os materiais granulares de base e sub-base usados nas estruturas de pavimentos são susceptíveis à degradação devido a vários fatores inerentes aos processos de produção, construção, e operação de estradas. 0 objetivo do presente artigo es quantificar a degradação de materiais granulares pela incidência da recompactação, usando para isto parâmetros de caracterização física e mecânica. Esta degradação foi avaliada em termos de mudanças em granulometria e resposta mecânica (i.e., CBR, parâmetros obtidos do ensaio triaxial estático, e módulo de resiliência). Os resultados obtidos sugerem que a compactação sucessiva levou à produção de partículas finas, atingindo um incremento do peso unitário seco, enquanto a resposta mecânica é reduzida. Reduções de CBR e modulo de resiliência são evidenciadas desde a primeira compactação mostrando o efeito da aplicação repetida de energia mecânica. Recomenda-se investigação adicional para validar a relação entre a degradação induzida pela recompactação e a gerada em campo.

Palavras-chave: agregados, subbase granular, degradação, ralação de suporte de Califórnia (CBR), módulo de resiliência, recompactação.

\section{Introducción}

Los materiales granulares para capas estructurales de pavimentos-bases y subbases granulares-se solían considerar materiales "inertes", que no sufrían daños substanciales a lo largo del tiempo (Tamayo et al., 1983). Sin embargo, investigaciones en el tema (Tamayo et al., 1983; Vallejo et al., 2006; Zeghal, 2009) demostraron que estos materiales estructurales son susceptibles a degradarse debido a múltiples factores inherentes a los procesos de producción, construcción y operación vial. Según Tamayo et al. (1983) existen dos tipos de degradación: la 
química que es la descomposición de los minerales que conforman los agregados por acción de agentes externos, y la mecánica que corresponde a los procesos que modifican las propiedades físicas debido a la aplicación de cargas sobre el material. La principal característica de ésta última es la producción de partículas finas a partir de la trituración de las partículas más gruesas en un material.

El proceso de compactación induce degradación físico-mecánica (Aughenbaugh et al., 1966; Tamayo et al., 1983). De hecho, este proceso induce los mayores cambios en el material granular alterando su comportamiento y acelerando su degradación (Aughenbaugh et al., 1966). En este sentido, un estudio previo (Zeghal, 2009) sobre bases granulares compactadas en campo y luego sometidas a pruebas de laboratorio para verificar su calidad, reportó cambios en su granulometría que afectaron la rigidez debido a la producción de arenas. Adicionalmente, Bábic et al. (2000) reportaron cambios en el material que conllevaron a la reducción de la permeabilidad y la capacidad de carga asociadas a la existencia de contenidos de arcilla superiores al 5\%. A nivel más general, en procesos de degradación por meteorización física de rocas (i.e., rotura sin cambios químicos), se presenta incremento del área específica y reducción del tamaño de partículas (Mitchell and Soga, 2005).

Rondón y Reyes, (2007) presentan un estado del arte de los estudios del comportamiento resiliente de los suelos granulares, en el que se discute que la granulometría, la cantidad de finos y la angularidad de las partículas tienen influencia en los valores de módulo resiliente de los suelos granulares. En lo que muchos autores están de acuerdo es en que existe un contenido óptimo de finos en los materiales a usarse como capas estructurales de un pavimento, por encima del cual, la respuesta mecánica de estos materiales tiende a disminuir.

De otra parte, a partir de pruebas realizadas en diferentes suelos se han establecido modelos matemáticos para predecir la rotura total esperada de partículas bajo una carga específica con el fin de definir el grado en el que la producción de partículas finas influye en el comportamiento de las capas de pavimento (Hardin, 1985). Adicionalmente, empleando simulación numérica, Vallejo et al. (2006) concluyeron que se presenta degradación mecánica de materiales granulares sometidos a cargas dinámicas y estáticas, caracterizada por la producción de partículas finas en las capas estructurales de un pavimento flexible. Según estas simulaciones, la trituración se presenta desde las capas superiores hacia las capas inferiores, con mayor incidencia durante las primeras aplicaciones de carga.

A partir de estas aproximaciones se busca caracterizar el daño ocasionado por la aplicación de cargas y avanzar en el desarrollo de herramientas y criterios que permitan mejorar la cuantificación del deterioro de los materiales granulares, y así mejorar los criterios para su selección y consecuentemente el diseño de estructuras de pavimento. No obstante, aún se requieren estudios adicionales sobre la susceptibilidad de los materiales granulares a la degradación y su efecto en la respuesta mecánica de los mismos para avanzar en la incorporación de este fenómeno a nivel del diseño estructural de pavimentos.

En dicho contexto, el objetivo del presente artículo es cuantificar la degradación que presentan materiales empleados como capas de subbase granular de pavimentos. Para este fin se evalúan los cambios en: (i) granulometría y (ii) respuesta mecánica-determinada en términos de la relación de soporte de California (CBR), además de parámetros obtenidos a partir del ensayo triaxial estático, y del módulo resiliente-de materiales granulares sometidos a procesos sucesivos de compactación. La compactación sucesiva (i.e., generada mediante compactación Proctor; aplicación de energía por impacto) se adoptó como mecanismo para inducir deterioro en los materiales de subbase y de esta forma obtener una primera aproximación al daño generado por: $(i)$ procesos de recompactación en campo o por aplicación excesiva de energía de compactación en campo, y (ii) las cargas repetidas de tránsito. Se reconoce así la necesidad de una fase futura de investigación 
para validar la relación entre la degradación inducida por recompactación en laboratorio y la generada en los materiales al ser empleados como capas estructurales de pavimentos en servicio.

\section{Materiales y métodos}

La Figura 1 esquematiza la metodología que se siguió para el desarrollo del proyecto. El estudio inició con la identificación de los materiales granulares de trabajo, su evaluación de calidad y la determinación de sus parámetros de compacta- ción. Posteriormente, se evaluaron los materiales en condición original y después de inducir degradación a través de un proceso de recompactación (o compactación sucesiva; Figura 1). En este proceso, un mismo espécimen fue compactado sucesivamente y después de cada compactación se evaluaron los cambios en su granulometría y respuesta mecánica, en términos de: (i) CBR, (ii) ensayo triaxial estático, y (iii) módulo resiliente. El estudio finalizó con la organización de resultados y el análisis del comportamiento de los materiales frente al proceso de compactación sucesiva.

\section{Figura 1. Esquema de la metodología de trabajo}

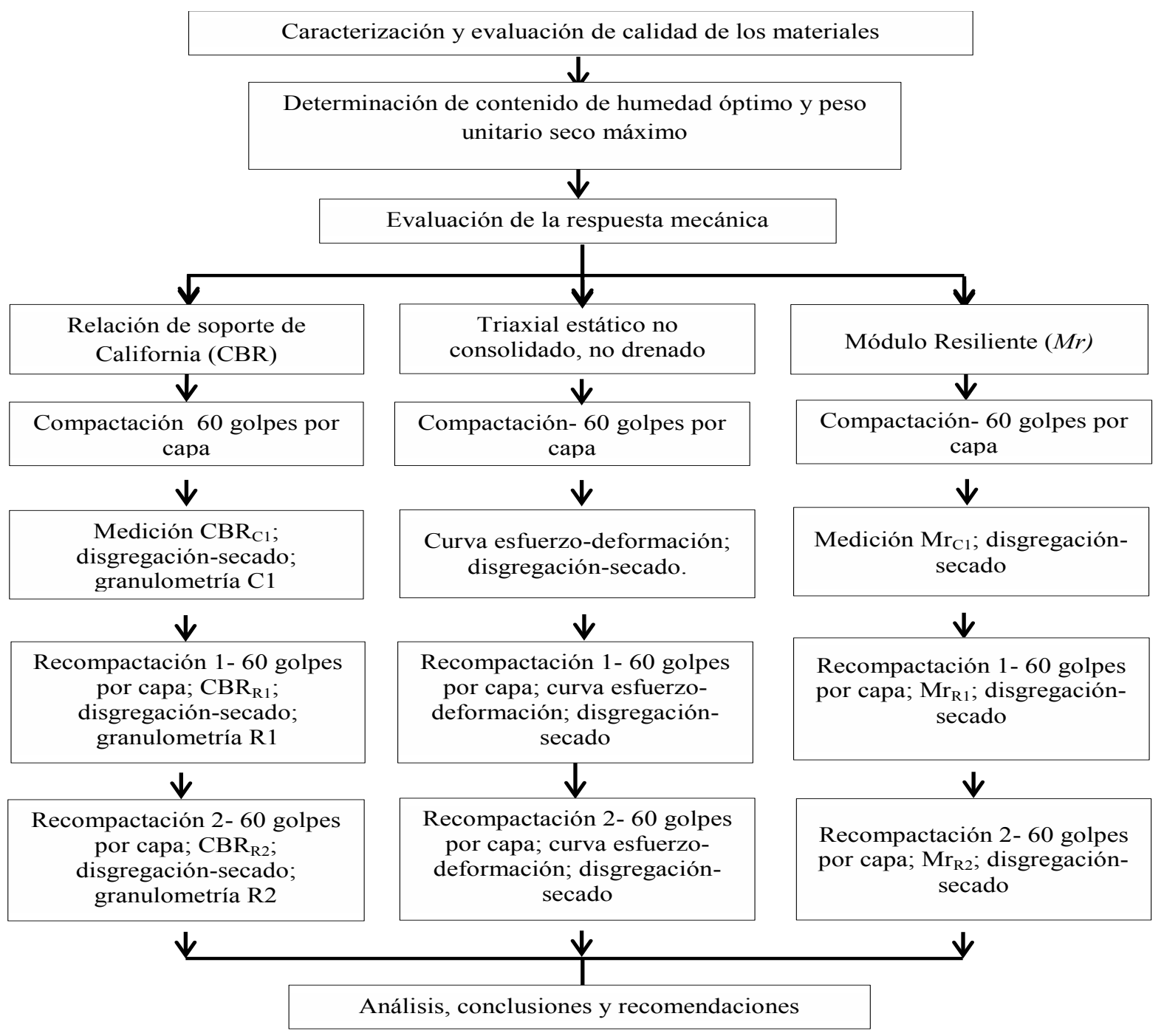




\subsection{Identificación de materiales de trabajo}

Las cuatro subbases granulares estudiadas provienen de diversas fuentes de materiales de construcción vial localizadas en los departamentos de Magdalena (SBG1), Bolívar (SBG-2 y SBG-4), y La Guajira (SBG3) en la zona norte de Colombia. Las SBG1, -2 y -4 provienen principalmente de depósitos tipo aluvión del periodo cuaternario, rocas sedimentarias de composición carbonatada (i.e., Caliza). El material granular SBG-3 está compuesto predominantemente de rocas ígneas plutónicas de composición intermedia (i.e., Andesitas), provenientes también de depósitos aluviales (Gómez et al., 2007).

\subsection{Evaluación de la calidad de los} materialesyparámetros decompactación

Conforme a las Especificaciones Generales de Construcción de Carreteras (i.e., artículo 320-13) del Instituto Nacional de Vías (INVIAS) (INVIAS, 2013), los materiales evaluados cumplieron con el huso granulométrico correspondiente a una subbase granular clase 2 .

Los ensayos de evaluación de calidad de material granular se realizaron según las normas de ensayo INVIAS (INVIAS, 2013), y se compararon con los requisitos establecidos en el Artículo 320-07 de las especificaciones INVIAS para subbases granulares con nivel de tránsito 3 (NT-3) (INVIAS, 2013). Los resultados correspondientes se presentan en la Tabla 1. Adicionalmente, la humedad óptima de compactación y el peso unitario seco máximo se determinaron a partir del ensayo Proctor modificado, método D según la norma I.N.V. E142-07 (INVIAS, 2013) y los resultados correspondientes se presentan en la Tabla 1.

\subsection{Evaluación de cambios en la dis- tribución granulométrica}

La evaluación de los cambios en la granulometría de los materiales debido al proceso de recompactación se cuantificó empleando el índice de quiebra de Marsal, $B_{g}$ (Muniz de Farias et al., 2013). Este índice es igual a la sumatoria de las diferencias en el porcentaje retenido en los $k$ tamices evaluados, antes de la compactación, $W_{K}^{i}$, y después de compactado, $W_{K}^{f}$, incluyendo sólo los valores positivos (Ecuación 1).

$$
B_{g}=\sum_{K}\left\langle W_{K}^{i}-W_{K}^{f}\right\rangle
$$

En este caso, el índice muestra la variación en los tamaños de las partículas, en puntos porcentuales, a partir de la diferencia en el porcentaje retenido en cada tamiz al aplicar el proceso de compactación sucesiva.

\section{TABLA 1. RESULTADOS DE ENSAYOS DE CARACTERIZACIÓN Y EVALUACIÓN DE CALIDAD}

\begin{tabular}{|c|c|c|c|c|c|c|c|c|c|}
\hline \multirow{2}{*}{ Material } & \multirow{2}{*}{$\begin{array}{c}\text { DMA } \\
\text { (\%) }\end{array}$} & \multirow{2}{*}{$\begin{array}{c}\text { DMD } \\
\text { (\%) }\end{array}$} & \multirow{2}{*}{$\begin{array}{l}\text { CA } \\
\text { (\%) }\end{array}$} & PS (\%) & \multirow{2}{*}{ LL (\%) } & \multirow{2}{*}{$\begin{array}{l}\text { IP } \\
\text { (\%) }\end{array}$} & \multirow{2}{*}{$\begin{array}{l}\text { EA } \\
\text { (\%) }\end{array}$} & \multirow{2}{*}{$\begin{array}{l}\text { Wo } \\
(\%)\end{array}$} & \multirow{2}{*}{$\begin{array}{c}Y_{\mathrm{dmmax}} \\
\left(t / \mathbf{m}^{3}\right)\end{array}$} \\
\hline & & & & $\mathrm{Na}_{2} \mathrm{SO}_{4}$ & & & & & \\
\hline SBG-1 (Ciénaga) & 46,7 & 26,6 & $11,1^{*}$ & 10,9 & 27,1 & NP & 25,1 & 8,6 & 2,17 \\
\hline SBG-2 (Cartagena) & 28,6 & 10,1 & $5,8^{*}$ & $12,6^{*}$ & 16 & 2,5 & $19,7^{*}$ & 7,8 & 2,06 \\
\hline SBG-3 (Riohacha) & 23,3 & 11,1 & $10,0^{*}$ & 11,3 & NP & NP & 51,7 & 8,1 & 2,18 \\
\hline SBG-4 (Cartagena) & 31,6 & 6,4 & $3,1^{*}$ & 3,5 & NP & $\mathrm{NP}$ & 63 & 10,3 & 2,06 \\
\hline Valor admisible (NT3) & $\leq \mathbf{5 0}$ & $\leq \mathbf{3 0}$ & $\leq 2$ & $\leq 12$ & $\leq \mathbf{2 5}$ & $\leq 6$ & $\geq 25$ & & \\
\hline
\end{tabular}

Nota: $\mathrm{DMA}$ = desgaste en la máquina de Los Ángeles; $\mathrm{DMD}=$ Desgaste en el equipo microdeval; $\mathrm{CA}=$ contenido de terrones de arcilla y partículas deleznables; PS = pérdida en ensayo de sanidad; $L L$ = límite líquido; IP = índice de plasticidad; $\mathrm{EA}=$ equivalente de arena; $\mathrm{NP}=$ no presenta; $\mathrm{Wo}=$ humedad óptima de compactación; $\gamma_{\mathrm{d} \text { máx }}=$ peso unitario seco máximo; NT = nivel de tránsito; ${ }^{*}=$ no cumple con la especificación INVIAS. 


\subsection{Evaluación de cambios en la res- puesta mecánica}

Los cambios en la respuesta mecánica de los materiales se evaluaron con base en los ensayos de CBR (norma I.N.V. E-148-13), triaxial estático no consolidado-no drenado (norma I.N.V. E-153-13) y módulo resiliente (norma I.N.V. E-156-13) (INVIAS, 2013).

Para la realización de ensayos triaxiales estáticos no consolidados no drenados, se tamizó el material SBG-1 y SBG-3 sobre el tamiz de $12.7 \mathrm{~mm}$ con el fin de cumplir con el tamaño máximo de partícula establecido en la norma I.N.V. E-153-07. Luego se fabricaron probetas cilíndricas compactadas, de 7.2 $\mathrm{cm}$ de diámetro por $14.4 \mathrm{~cm}$ de altura. La compactación del material se hizo con el martillo Proctor estándar, en 5 capas, aplicando 60 golpes/capa en un molde metálico diseñado especialmente para ello. En las probetas para ensayo triaxial se repro- dujo el peso unitario seco establecido mediante el ensayo Proctor modificado. Una vez compactadas, se extrajeron las probetas del molde utilizando un extractor horizontal de muestras e inmediatamente después fueron sometidas al ensayo triaxial estático. Los parámetros evaluados en este ensayo se definen en la Tabla 2.

Los ensayos de CBR, módulo resiliente y triaxial estático se llevaron a cabo a la humedad óptima de compactación $\left(W_{o}\right)$ de cada subbase granular, compactadas a los pesos unitarios secos reportados en la Tabla 1. Adicionalmente, se realizaron mediciones de CBR a humedad de saturación $\left(W_{s}\right)$ (i.e., después de cuatro días de inmersión) y se ejecutó el ensayo triaxial estático sobre probetas saturadas-en el equipo triaxial-para cuantificar el efecto del incremento de humedad sobre la respuesta mecánica de los materiales.

\section{TABLA 2. PARÁMETROS EVALUADOS EN EL ENSAYO TRIAXIAL ESTÁTICO}

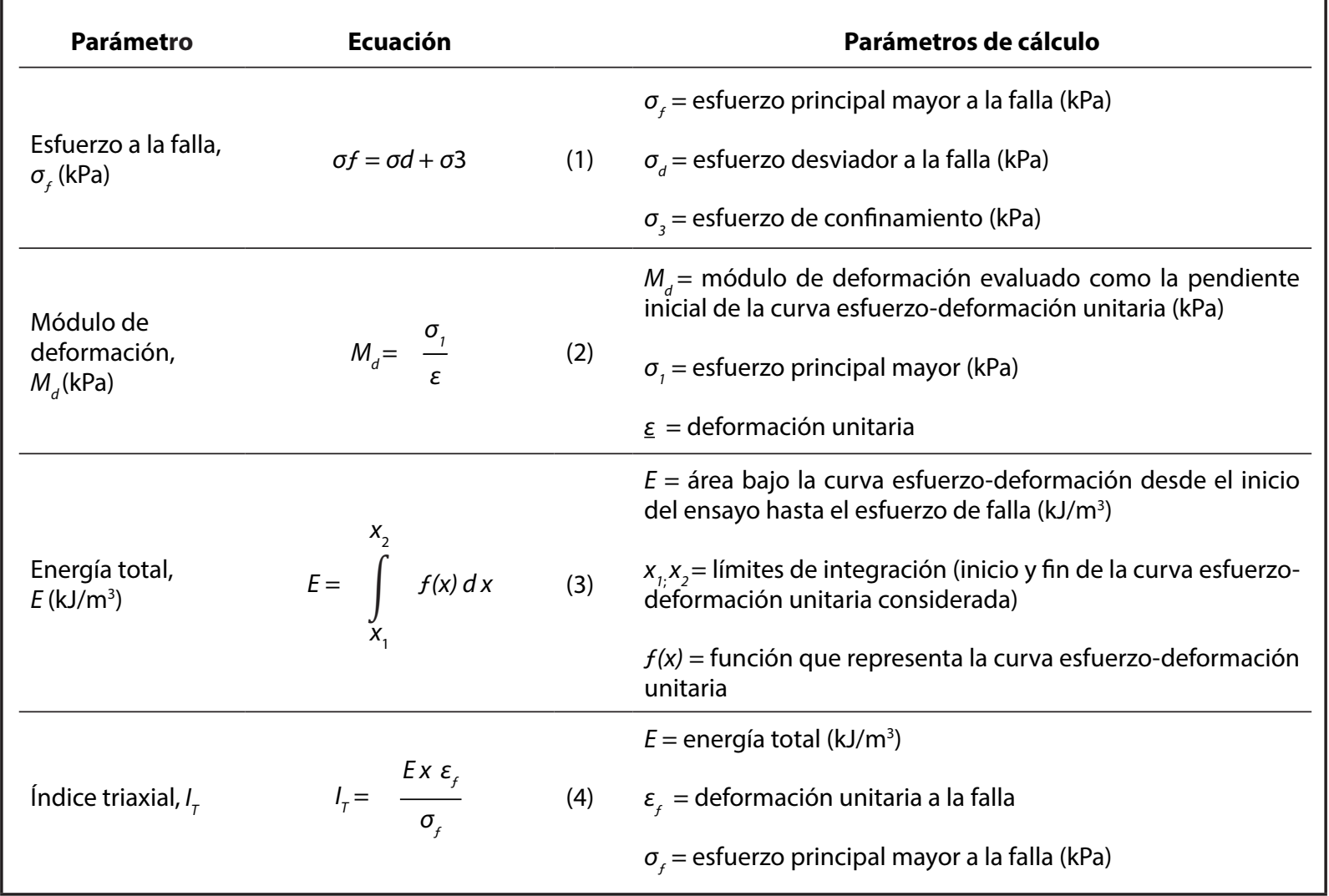


Figura 2. Distribución granulométrica de subbases granulares sujetas a recompactación

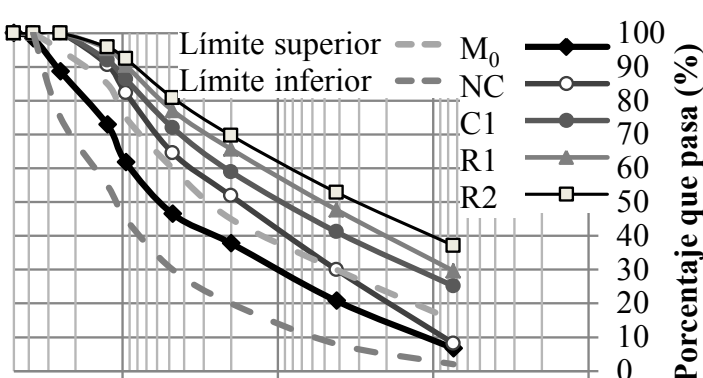

10

Tamaño (mm)

(a) SBG-1

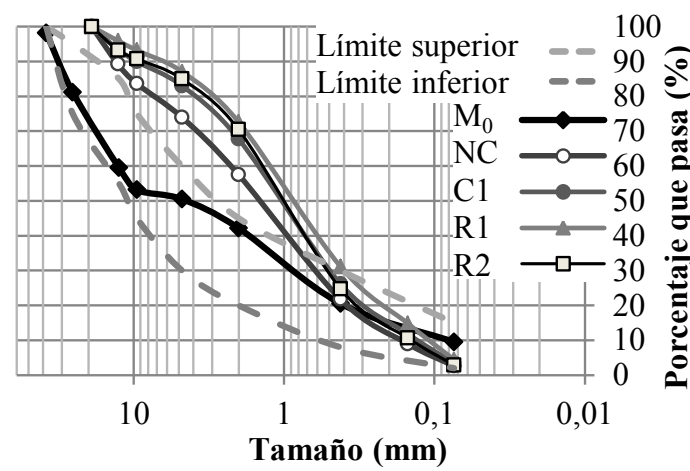

(c) SBG-3

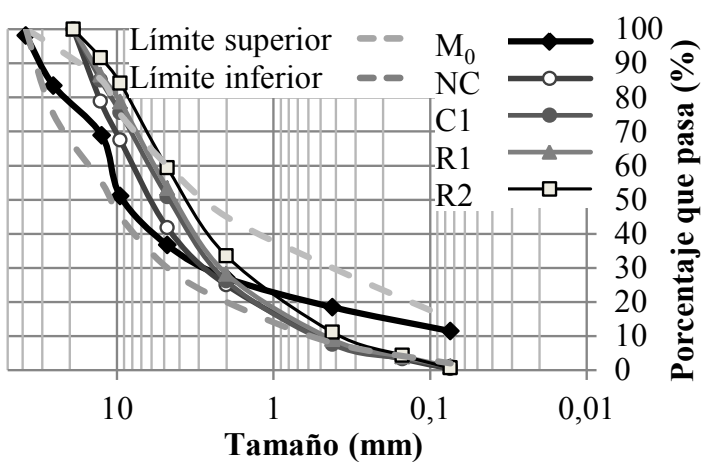

(b) SBG-2

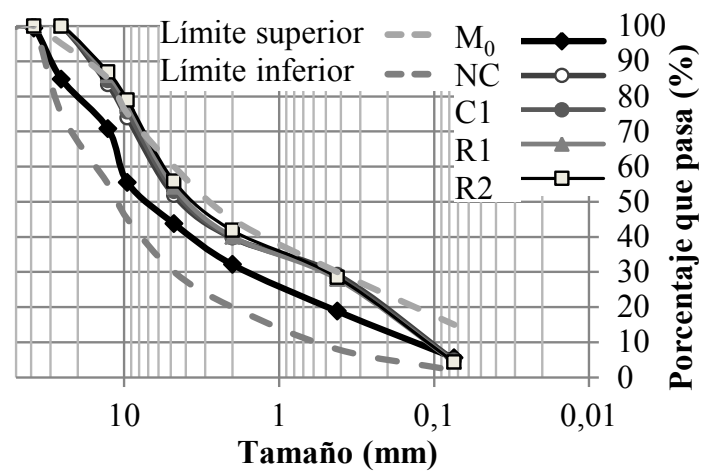

(d) SBG-4

\section{Resultados y análisis}

Esta sección presenta los resultados de la evaluación del efecto de la recompactación en subbases granulares utilizando indicadores de comportamiento físico (i.e., cambios en la granulometría) y mecánico (i.e., cambios en CBR, indicadores calculados a partir del ensayo triaxial estático, y módulo resiliente).

\subsection{Cambios en la distribución gra- nulométrica}

La Figura 2 presenta las curvas granulométricas de las subbases estudiadas en su condición " $\mathrm{M}_{0}$ " (material original muestreado en cantera), "NC" (material preparado para ensayo de CBR, no compactado), "C1" (material evaluado después de compactado por primera vez para medición convencional de CBR), y "R1" y "R2" (material evaluado después de ser recompactado una y dos veces, res- pectivamente, como probeta para el ensayo de CBR). Adicionalmente, la Figura 3 presenta los resultados del índice de quiebra de Marsal con el cual se cuantifican los cambios inducidos en la granulometría por el proceso de recompactación. Valores pequeños del índice muestran materiales con baja degradación por el proceso de recompactación.

\section{Figura 3. Índice de quiebra de Marsal}

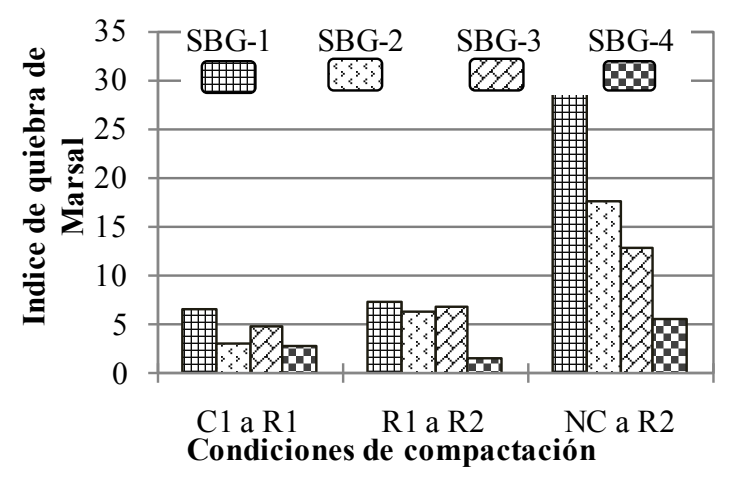


En general, el efecto de la aplicación de energía mecánica sobre la distribución de tamaños de los materiales estudiados corresponde a la producción de arenas y finos (i.e., material pasa tamiz 200). Este efecto se da incluso como resultado de la primera compactación, necesaria para la ejecución convencional del ensayo de CBR (Figura 2). Específicamente, la SBG-1 presenta un aumento de finos considerable, que se exhibe en el incremento hasta casi un $40 \%$ del material que pasa el tamiz 200. En los otros materiales se observa que hay una variación en los porcentajes de grava y arena, pronunciándose con mayor énfasis en la SBG-3, donde existe un aumento notable en la cantidad de arenas. Adicionalmente, la SBG-4 corresponde al material de menor susceptibilidad a modificar su granulometría por el proceso de recompactación, mientras en el extremo opuesto se identifica la SBG-1, que presenta mayor susceptibilidad.

Las discrepancias indicadas entre los materiales evaluados se relacionan con la resistencia intrínseca de sus partículas. De las subbases ensayadas, la SBG-4 es la que presenta mejores resultados en el ensayo de desgaste en el equipo microdeval (Tabla 1), mientras la SBG-1 muestra los resultados más desfavorables y las SBG-2 y SBG-3 exhiben resultados intermedios, lo que coincide con la susceptibilidad a la variación en la distribución granulométrica presentada en la Figura 2 y con los valores obtenidos en la Figura $\mathbf{3}$ con el índice de quiebra Marsal. Sin embargo, esta relación no se conserva al analizar los ensayos de desgaste en la máquina de Los Ángeles, ya que la SBG-4 presenta valores altos en este indicador en comparación con los otros materiales (Tabla 1).

Adicionalmente, las características geométricas de las partículas podrían estar asociadas-al inducir variación en la microestructura de las probetas compactadas-con las diferencias en la degradación exhibida por los materiales evaluados. Sin embargo, la evaluación de estas características se encuentra fuera del alcance del presente estudio.

\subsection{Cambios en la respuesta mecáni- $\mathrm{Ca}-\mathrm{CBR}$}

En las Figuras $\mathbf{4 a}$ y $\mathbf{4 b}$ se muestran los valores individuales de las réplicas ejecutadas (i.e., MI: espécimen 1, MII: espécimen 2, y MIII: espécimen 3) del ensayo de CBR y sus correspondientes valores de peso unitario seco. Adicionalmente, en las Figuras 4c y 4d se presentan los valores promedio de CBR y peso unitario seco obtenidos a partir de las réplicas antes indicadas. Estos valores de CBR se presentan para probetas evaluadas en condición de humedad óptima (0) y de saturación (S) (i.e., después de inmersión por cuatro días) para las diferentes condiciones de compactación evaluadas (i.e., C1, R1 y R2).

En los cuatro materiales estudiados se obtuvo una disminución en el CBR al ser sometidos a compactación repetida, lo que refleja la degradación producto de la aplicación sucesiva de energía mecánica. Al asociar esta respuesta con la granulometría, se observó que pequeños cambios en la granulometría inducen grandes variaciones en la respuesta mecánica en términos de capacidad de soporte, como es notable en las SBG-2 y SBG-4. Con base en los valores promedio de CBR (Figuras $\mathbf{4 c}$ y $\mathbf{4 d}$ ) se puede inferir que las subbases reportan decrementos cercanos al $50 \%$ en la respuesta mecánica entre C1 y R1, evidenciándose que la segunda compactación indujo la mayor pérdida de respuesta mecánica al material. De hecho, si bien el comportamiento de cada material ante la recompactación es diferente (Figuras 4c y 4d), después de la primera recompactación (R1) las cuatro subbases estudiadas no alcanzaron la especificación de CBR mínimo establecida por INVIAS (i.e., CBR $>40 \%$ para una subbase clase A con NT-3) (INVIAS, 20013).

Adicionalmente, después de inducir el proceso repetido de compactación, inmersión y disgregación hasta alcanzar la recompactación 2 , en condiciones de humedad optima de compactación, se presenta disminución de la respuesta mecánica del $85 \%$ en la SBG-1, cerca del 58\% para el material SBG-2, en un $72 \%$ para la SBG-3 y de un 66\% para la SBG4. De 
igual manera, con excepción de la SBG-1, se obtuvo un aumento del peso unitario seco al recompactar los materiales. Este incremento se debe a la disminución de la proporción de partículas tamaño grava y la producción de arenas y finos (Figura 2) que permite mayor empaquetamiento de sólidos por unidad de volumen. Por otro lado, para la SBG-1 se presentó disminución del peso unitario seco, lo cual puede deberse a que la alta cantidad de finos producidos por la recompactación dificulta la obtención de altos pesos unitarios debido a la uniformidad de tamaños de partícula que impiden un mejor empaquetamiento.

\section{Figura 4. Relación entre CBR y peso unitario seco en términos de valores individuales (réplicas) (a) y (b) y valores promedio} (c) y (d).

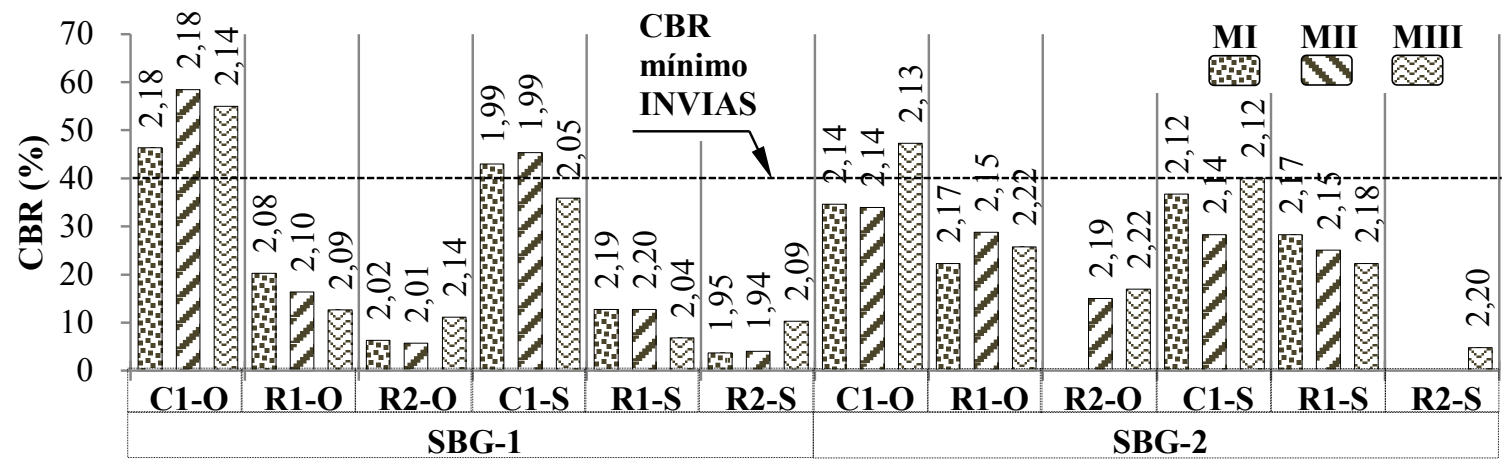

(a)

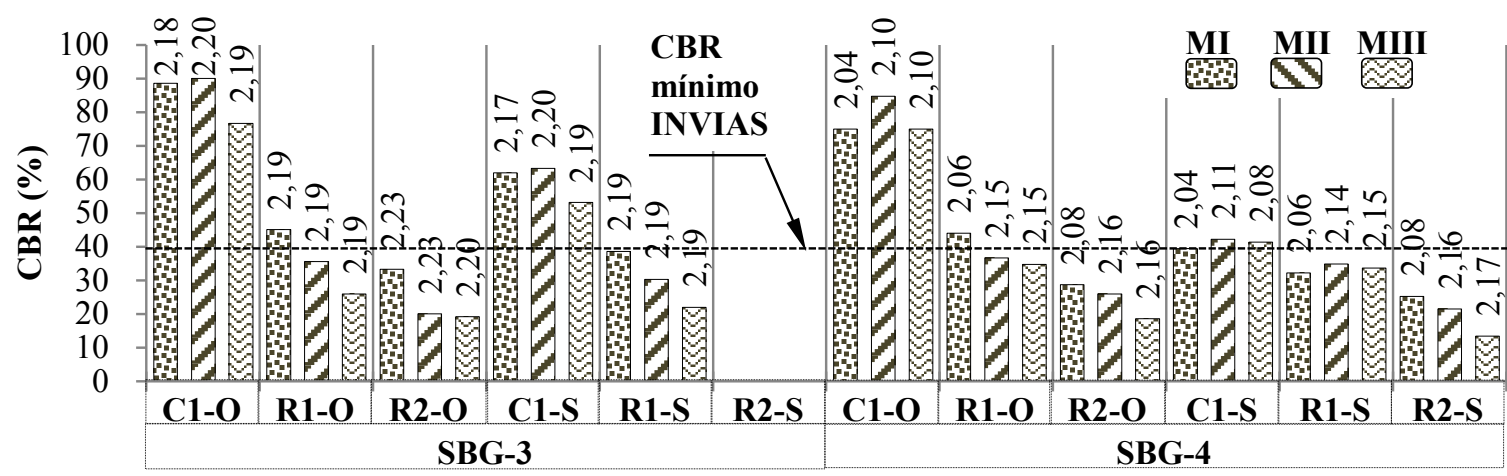

(b)

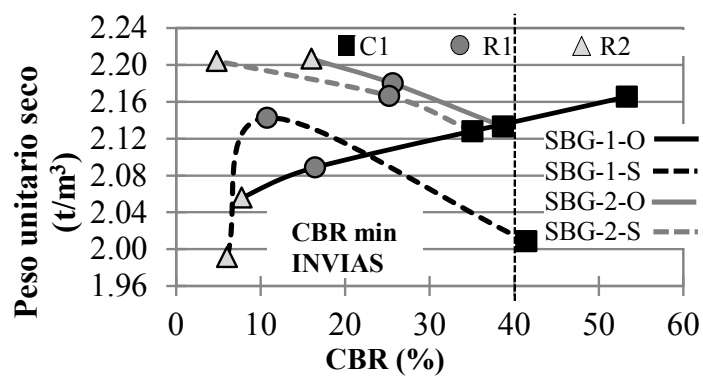

(c)

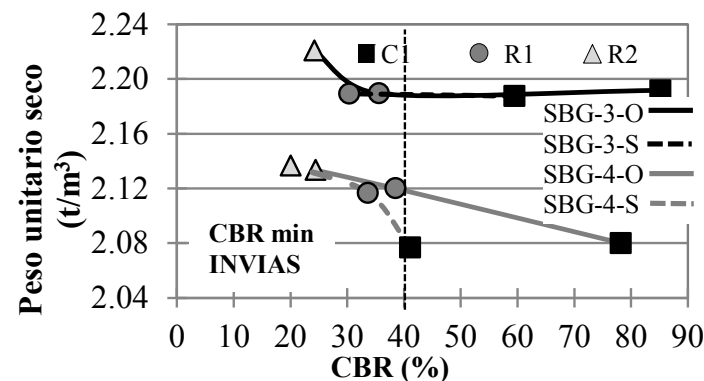

(d)

Nota: MI: muestra 1 ; MII: muestra 2; MIII: muestra 3; O: humedad óptima; S: humedad de saturación 


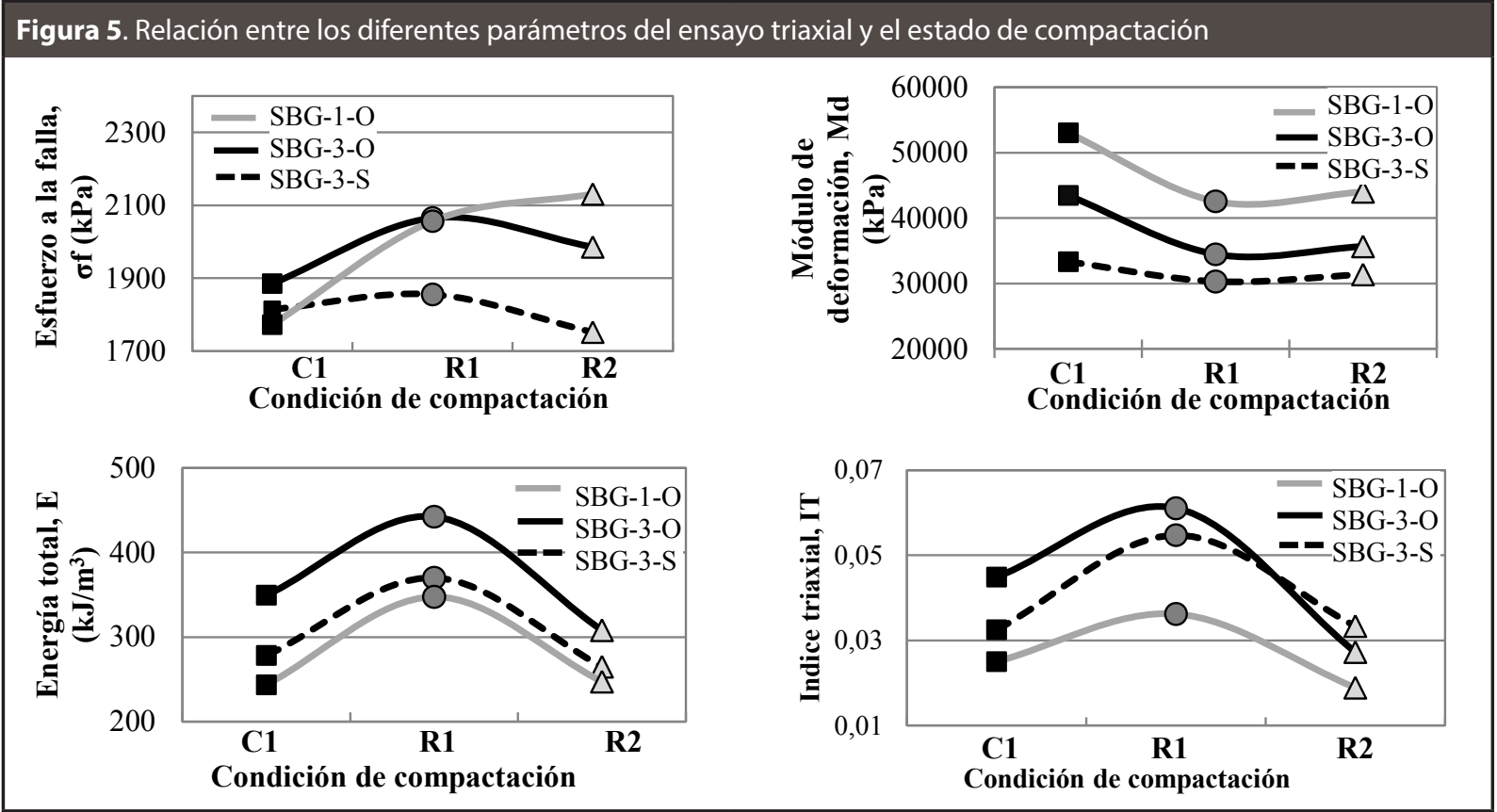

Por otra parte, se observa la pérdida de respuesta mecánica ocasionada por la saturación del material (Figuras 4c y 4d). El contenido de humedad del suelo condiciona la succión que desarrolla. Ésto se debe al cambio de las fuerzas capilares, en los meniscos que forman entre sí las partículas, asociado a los cambios de humedad. Así, altos contenidos de humedad-altos niveles de saturación-se asocian con bajos valores de succión, lo que a su vez se refleja en una disminución en la respuesta mecánica del material granular (i.e., rigidez y resistencia a deformación permanente). De esta forma, y según las expectativas teóricas, bajo las mismas condiciones de compactación se observa que a la humedad óptima de compactación los valores de CBR son mayores que aquellos registrados bajo condiciones de saturación.

\subsection{Cambios en la respuesta mecá-} níca - ensayo triaxial estático

La Figura 5 presenta los resultados de los ensayos triaxiales estáticos realizados a probetas de los materiales SBG-1 y SBG-3. La Figura 5a denota el incremento en el esfuerzo a la falla en las probetas de la SBG-3 entre sus estados C1 y R1 y una disminución entre sus estados R1 a R2, mientras que para la SBG-1 aumenta desde C1 hasta R2. En cuanto al módulo de deformación (Figura 5b) se aprecia un decremento al pasar la probeta de estado C1 a R1 y luego un incremento del mismo al pasar de estado R1 a R2, pero sin alcanzar los niveles de la condición C1. En las Figuras $\mathbf{5 c}$ y $\mathbf{5 d}$ se puede notar, respectivamente, que debido al proceso de recompactación la energía total y el índice triaxial presentan un incremento significativo al pasar la probeta de estado C1 a R1, y luego presentan una disminución significativa al llegar la probeta a la condición R2.

Los resultados anteriores indican el cambio de la respuesta de las subbases SBG-1 y SBG-3 debido al efecto de los procesos de recompactación. De hecho, la disminución del módulo de deformación-adoptado como un indicador de rigidez del material—es coincidente con la disminución de módulo resiliente (tal como se discute a continuación). Sin embargo, los índices restantes evaluados a partir del ensayo triaxial evidencian una respuesta más favorable en la condición R1 que en las condiciones C1 y R2. En principio, estos resultados contradicen la degradación evidenciada a partir de los ensayos de CBR y 
módulo resiliente y sugieren que el comportamiento del material, evaluado en términos de energía y resistencia al corte, puede diferir del evaluado en ensayos de carga repetida (i.e., módulo resiliente) y falla por penetración del material (i.e., CBR).

\subsection{Cambios en la respuesta mecáni- ca - módulo resiliente}

La Figura 6 presenta la relación entre el esfuerzo de confinamiento- $\theta$ : sumatoria de los esfuerzos principales-y el módulo resiliente $\left(M_{r}\right)$ para las subbases granulares sometidas a compactación sucesiva. Las variaciones presentadas en el módulo resiliente de las subbases granulares ensayadas se asocian principalmente a dos factores: las características propias del material y componentes externos (Lekarp et al., 2000).

Las características propias que afectan el comportamiento de los materiales son, entre otras, la granulometría, actividad de los finos, la humedad, el tamaño máximo nominal y tipo de material (Lekarp et al., 2000). Según la caracterización de los materiales (Tabla 1), en términos de \%DMA y \%DMD, la SGB-3 presenta menores resultados; adicionalmente presenta menos PS(\%) y menor CA (\%) que la SBG1, lo que la hace menos susceptible a la degradación.

Lo anterior, es congruente con el comportamiento de las subbases ensayadas presentado en la
Figura 6, donde se evidencia que la susceptibilidad a la recompactación en la SBG-1, es mayor que la presentada en la SBG-3. Para la SBG-1 se obtienen disminuciones del $\mathrm{Mr}$ del orden de 23\% en R1 y $14 \%$ en R2. Para la SBG-3, la reducción en el $M r$ es del 15\% en la R1 y del 9\% en la R2.

El factor externo con mayor incidencia en el comportamiento del material es el esfuerzo de confinamiento (Transporte, 2001; Rondón \& Reyes, 2007). De hecho, las Figuras 6a y 6b muestran cómo el módulo resiliente es directamente proporcional al esfuerzo de confinamiento, independientemente de la condición de compactación que implica la pérdida progresiva de rigidez.

Adicional a esto, los resultados presentados en la Figura 6, muestran que a medida que se incrementa la compactación, el esfuerzo de confinamiento tiene una mayor incidencia en el $M r$, siendo más evidente en la etapa de $\mathrm{R} 2$, donde se presenta aumentos del $\mathrm{Mr}$ al incrementar el esfuerzo de confinamiento de 100 a $600 \mathrm{kPa}$. Con los incrementos mencionados, se obtiene un aumento del $\mathrm{Mr}$ del 15\% para la SBG-1 y del 21\% en la SBG-3. Este aumento en el $\mathrm{Mr}$ puede deberse a la producción de finos debido a la compactación y degradación del material natural, en materiales con mayor cantidad de finos el efecto del esfuerzo de confinamiento es mayor (Transporte, 2001).

\section{Figura 6. Relación entre esfuerzo de confinamiento y módulo resiliente para la SBG-1 (a) y la SBG-3 (b)}

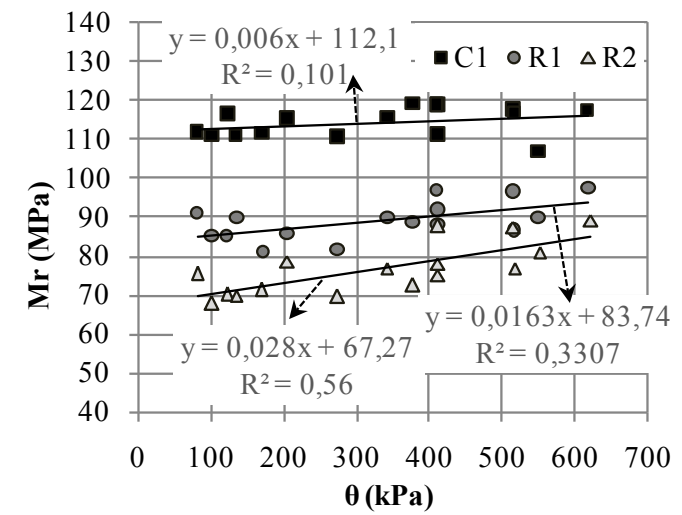

(a) SBG-1

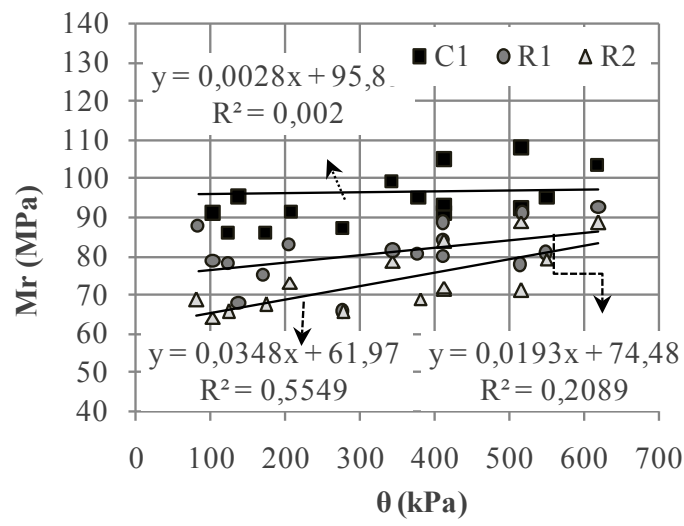

(b) SBG-3 


\section{Conclusiones y recomendaciones}

El presente estudio evaluó los efectos de procesos sucesivos de compactación-adoptados como mecanismo para inducir deterioro con el fin de obtener una primera aproximación al daño generado por: (i) procesos de recompactación en campo y (ii) las cargas repetidas de tránsito-de materiales granulares tipo subbase. A partir de los resultados obtenidos en el estudio se presentan las siguientes conclusiones:

- Los materiales estudiados se degradaron por compactación sucesiva, lo cual se evidenció cuantitativamente en los cambios de granulometría que conllevaron en general a la disminución de respuesta mecánica de los materiales. Sin embargo, la primera recompactación podría incidir positivamente en la resistencia al corte. Los cambios evidenciados se asocian probablemente con la modificación de la estructura interna que adquiere el material al aplicar energía mecánica sucesivamente-o en exceso durante el proceso de compactación en campo-. Sin embargo, se requiere investigación futura para cuantificar los posibles cambios en la estructura interna de los materiales.

- El peso unitario seco es utilizado actualmente como indicador de la calidad del proceso de compactación en campo. No obstante, se puede observar que sin importar la disminución de la respuesta mecánica de las SBG-2,-3 y -4, la tendencia del peso unitario seco fue mantenerse constante o en aumento. Por tanto, si se obtuviera un bajo peso unitario en campo, $y$ se decidiera disgregar el material para volver a compactarlo, aunque sea posible alcanzar el peso unitario seco especificado de campo, la pérdida en el desempeño de los materiales podría ser apreciable.

- Los resultados obtenidos motivan el desarrollo de investigación adicional sobre la degradación por efectos mecánicos de los materiales granulares para obtener indicadores que pue- dan ser incluidos a nivel de evaluación de calidad de materiales o de diseño estructural de pavimentos y de esta forma, permita mejorar la calidad de los pavimentos.

\section{Agradecimientos}

Los autores expresan sus agradecimientos a Ángela Torres, David Crespo y Tatiana Jiménez, estudiantes de Ingeniería Civil de la Universidad del Magdalena, por el apoyo brindado en la ejecución parcial de ensayos de laboratorio y al ingeniero geólogo Jorge Corrales por su asesoría en la descripción geológica de los materiales. Igualmente, se agradece el apoyo brindado por la Vicerrectoría de Investigación, a través de FONCIENCIAS, y la colaboración del personal del Laboratorio Integrado de Ingeniería Civil-LIIC de la Universidad del Magdalena.

\section{Referencias}

AUGHENBAUGH, N. B.; JOHNSON, R. B.; and YODER, E. J. (1966). "Degradation of base course aggregates during compaction". Purdue Research Foundation Lafayette In. Laboratory Technical Report 166.

BABIC, B.; PRAGER, A.; and RUKAVINA, T. (2000) "Effect of fine particles on some characteristics of granular base courses". Materials and Structures, vol. 33, pp. 419-424.

GÓMEZ, J.; NIVIA, A.; MONTES, N. E.; JIMENEZ, D. M.; TEJADA, M. L.; SEPULVEDA, M. J.; OSORIO, J. A.; GAONA, T.; DIEDERIX, H.; URIBE, H.; and MORA, M. (2007), Mapa geológico de Colombia. INGEOMINAS, Bogotá D.C.

HARDIN, B. (1985). "Crushing of soil particles". Journal of Geotechnical Engineering, vol. 111, núm. 10, pp. 1177-1192.

INSTITUTO MEXICANO DEL TRANSPORTE (2001). Módulos de Resiliencia en Suelos Finos y Materiales Granulares. Publicación Técnica núm, 142, Sanfandila, Qro, México.

INVIAS(2013). Especificaciones generales de construcción de carreteras y normas de ensayo para carreteras. Bogotá D.C.

LEKARP, F.; ISACSSON, U.; and DAWSON, A. (2000). "State of the art. I: Resilient response of unbound aggre- 
gates". Journal of Transportation Engineering, vol. 126, pp. 66-75.

MITCHELL, J. K.; and SOGA, K. (2005). Fundamentals of soil behavior, Hoboken, New Jersey,.

MUNIZ DE FARIAS, M.; GÓMEZ-JIMÉNEZ, A.; and QUIÑONES-SINISTERRA, F. (2013) . Uso de agregados reciclados en capas de base y revestimientos asfálticos. XIX Simposio Colombiano sobre Ingeniería de Pavimentos. Bogotá D.C.: pp. XXXX

RONDÓN , H y REYES, F. (2007). “Comportamiento resiliente de materiales granulares en pavimentos flexibles: estado del conocimiento". Revista Ingenierías, Universidad de Medellín., vol.6, num. 11, pp. 65-90

TAMAYO, J.; GUTIÉRREZ, C. I.; and CABRERA, J.( 1983). "Estudio de la degradación de los agregados pétreos durante la vida útil de los pavimentos". Revista Ingeniería e Investigación., vol. 2, núm. 1, pp. 13-21.

VALLEJO, L.; LOBO-GUERRERO, S.; and HAMMER, K. (2006). "Degradation of a granular base under a flexible pavement: Dem simulation". Internactional Journal of Geomechanics., vol. 6, núm. 6, pp. 435-439.

ZEGHAL, M. (2009). "The impact of grain crushing on road performance". Geotechnical and Geological Engineering., vol. 27, núm. 4, pp. 549-558.

\section{PARA CITAR ESTE ARTÍCULO / TO REFERENCE THIS ARTICLE / PARA CITAR ESTE ARTIGO /}

Álvarez Lugo, A.E.; Espinosa Ruiz, L.V.; Ortiz Rivera, P.A.; Hurtado Robayo, M.D.; Cotes De La Hoz, L.E.; López Esalas, Y.M. (2019). Evaluación de la degradación por compactación de materiales granulares tipo subbase. Revista EIA, 16(31), enero-junio, pp. 13-25. [Online]. Disponible en: https://doi. org/10.24050/reia.v16i31.746 